\title{
Evaluación de la descomposición de hojarasca en el remanente de bosque de la Universidad de lbagué
}

\section{Evaluation of leaf litter decomposition in the forest remnant of Universidad de Ibagué}

\author{
Adriana Lucia Oliveros Lozada' \\ Blanca Myriam Salguero Londoño²
}

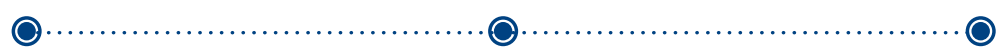

Recepción: 29/09/2021

Aprobación: 28/10/2021

Publicación: 22/12/2021

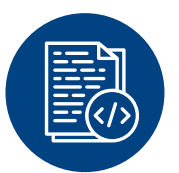

Para citar este artículo

Oliveros Lozada, A. L., \& Salguero Londoño, B. M. (2021). Evaluación de la descomposición de hojarasca en el remanente de bosque de la Universidad de Ibagué. Indagare, (9), 41-45.

https://doi.org/10.35707/indagare/904

\footnotetext{
1 Grupo de investigación Naturatu, Ibagué, Colombia. ORCID: 0000-0002-7574-873X. Correo electrónico: adriana.oliveros@unibague.edu.co

2 Grupo de investigación Naturatu, Universidad de Ibagué, Ibagué, Colombia. ORCID: 0000-0003-0508-8753.

Correo electrónico: blanca.salguero@unibague.edu.co
} 


\begin{abstract}
Resumen
Los bosques prestan diferentes servicios ecosistémicos importantes para las dinámicas naturales de estos ecosistemas, dentro de los cuales se encuentra la descomposición de la hojarasca. El objetivo del presente trabajo fue evaluar la tasa de descomposición de la hojarasca en el remanente del bosque El Espejo de la Universidad de Ibagué. Se evaluó la tasa de descomposición de la hojarasca en un periodo seis meses, en dos franjas de bosque. Se pudo establecer que la tasa de descomposición de hojarasca para el remanente del bosque El Espejo de la Universidad de Ibagué es media. La descomposición de la hojarasca se relaciona con la conservación de los suelos, lo cual permite que la microfauna contribuya a este proceso.
\end{abstract}

\begin{abstract}
Forests provide different ecosystem services important for the natural dynamics of these ecosystems, among which is the decomposition of leaf litter. The objective of this study was to evaluate the litter decomposition rate in the remnant of El Espejo forest at Universidad de Ibagué. The decomposition rate of leaf litter was evaluated over a period of six months, in two forest strips. It was established that the litter decomposition rate for the remnant of El Espejo forest at Universidad de Ibagué is average. Litter decomposition is related to soil conservation, which allows microfauna to contribute to this process.
\end{abstract}

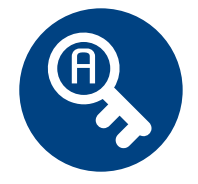

Palabras claves: Peso seco, bosque de ribera, peso remanente, servicios ecosistémicos.

Key words: Dry weight, riparian forest, remnant weight, ecosystem services.

\title{
1. Introducción
}

Los bosques subandinos son ecosistemas perturbados por la actividad antrópica generando cambios en el uso de sus suelos, debido al desarrollo de actividades agropecuarias, la deforestación, entre otras acciones que contribuyen a la disminución de flora y fauna pertenecientes a estos ecosistemas (Alvear \& Betancur, 2010). El remanente de bosque El Espejo de la Universidad de Ibagué se categoriza como un bosque subandino debido a características propias de dicho ecosistema, asimismo debido a que se encuentra ubicado en el margen de la quebrada Ambalá. También es considerado como un bosque de ribera y un bosque urbano porque se encuentra dentro del municipio de Ibagué.

Los bosques ribereños son ecosistemas que permiten la interacción de especies terrestres y acuáticas, además de esto, se menciona que contribuyen a la disminución 
de la erosión, así como la reducción de sustancias contaminantes y sedimentos, que pueden llegar a los cuerpos de agua; por otro lado, estos tipos de bosques son responsables de cambiar la composición de nutrientes en diferentes procesos químicos y biológicos (Ceccon, 2003; Naiman, Decamps, Mcclain, \& Likens, 2010).

Por su parte, los bosques urbanos son ecosistemas que se desarrollan en zonas aledañas a las comunidades urbanas (Moll \& Gangloff, 1987), estos cuentan con diferentes servicios ecosistémicos que benefician a las comunidades que los colindan (Balvanera, 2012), estos ecosistemas permiten regular la temperatura y las inundaciones y mejoran la calidad del aire en las ciudades (Díaz \& Curiel, 2012). Asimismo, regulan diferentes ciclos como lo son el aporte de nutrientes al suelo procedente de la hojarasca.

La hojarasca es considerada como uno de los principales elementos que aportan y retornan nutrientes al suelo, mediante procesos fundamentales como su producción y descomposición, los cuales contribuyen a la dinámica natural de los suelos (Isaac \& Nair, 2006). Debido a su importancia y a la poca información que se tiene frente a estudios similares en el bosque El Espejo de la Universidad de Ibagué, el objetivo del trabajo de investigación fue evaluar la descomposición de la hojarasca en el remanente del bosque El Espejo de la Universidad de Ibagué.

\section{Materiales y métodos}

Se seleccionaron dos franjas del remanente de bosque El Espejo de la Universidad de Ibagué, la franja uno se encuentra ubicada cerca a la quebrada Ambalá y presenta un grado alto de conservación y su flora es propia de bosques de ribera; en la franja dos se presenta un menor grado de conservación ya que se evidencia intervención antrópica y algunas de sus especies de flora han sido introducidas. Allí se recolectaron $20 \mathrm{~g}$ de hojarasca en bolsas de descomposición litterbags, estas fueron elaboradas manualmente con tamaño de 20 x $20 \mathrm{~cm}$, con malla de $0.5 \mathrm{~mm}$ de poro, lo cual permite el ingreso y la interacción de la microfauna del suelo dentro de las bolsas (Wider \& Lang, 1982).

Las bolsas fueron ubicadas sobre el suelo en línea de seis, luego se añadió una capa de biomasa para evitar afectaciones provenientes de la actividad del hombre. La recolección de cada bolsa se realizó de manera aleatoria mensualmente durante los seis meses del muestreo. Una vez recolectadas las bolsas se analizaron en el laboratorio de Biología de la Universidad de Ibagué. El material fue secado en un horno de aire marca Memmer, referencia UN 110 Plus a $60^{\circ} \mathrm{C}$ durante 48 horas hasta obtener peso constante, seguido a esto las bolsas fueron pesadas en una balanza analítica marca $A \& D$ modelo FX 2000-1, identificando la diferencia entre peso inicial y peso remanente.

Los datos obtenidos fueron analizados bajo pruebas no paramétricas de comparación de medias y grupos de análisis de regresión lineal en el software Infostat versión profesional. La tasa de descomposición se evaluó por medio de un análisis varianza no paramétrico de Kruskal Wallis.

\section{Resultados}

El porcentaje de peso remanente durante los seis meses del muestreo (180 días) fue en promedio 25,36 \% en el remanente de bosque El Espejo de la Universidad de Ibagué. Para la franja uno el porcentaje de peso remanente fue de $27,33 \%$ y en la franja dos fue de $23,38 \%$ (Figura 1). 
Figura 1. \% Peso remanente por franjas a lo largo del muestreo

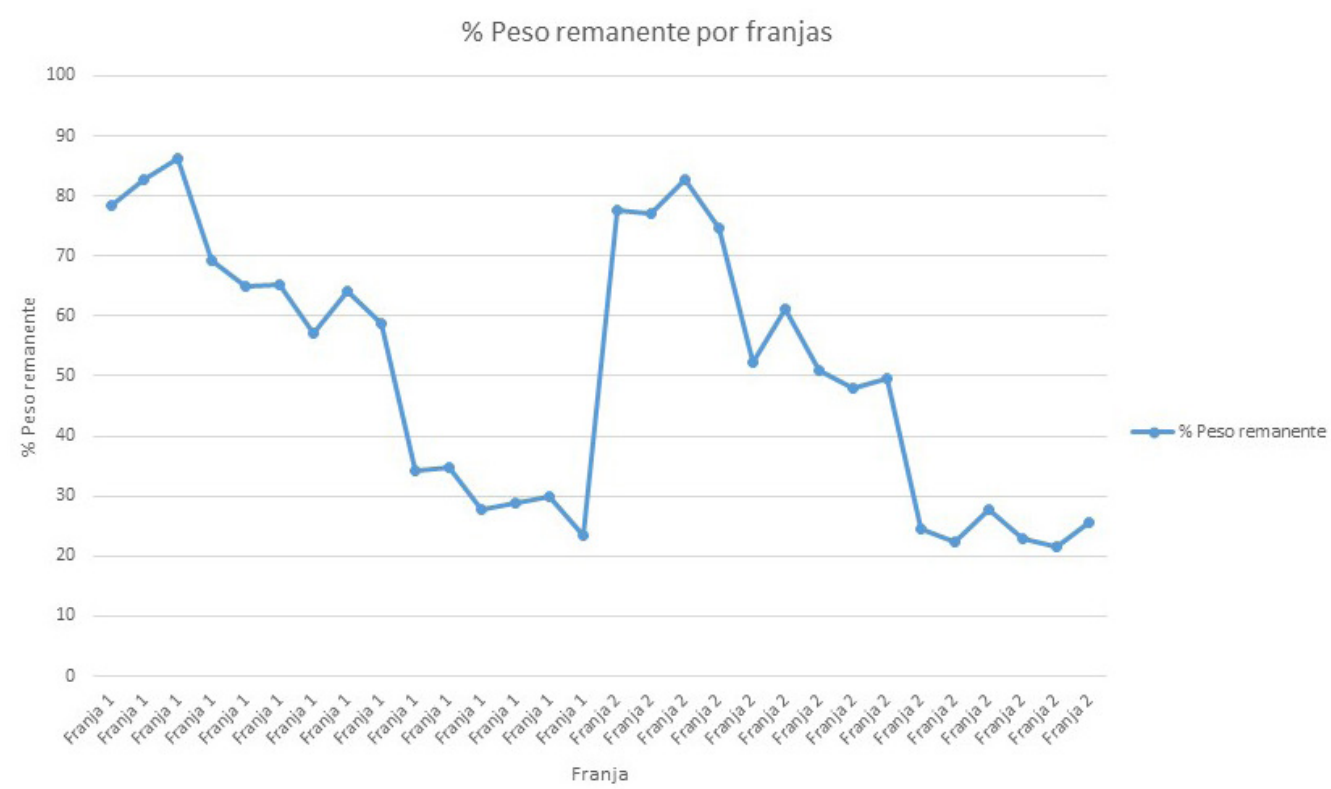

Fuente: Elaboración propia

La tasa de descomposición promedio de $k=0,0077$ en la franja uno la tasa de descomposición $(k) \mathrm{f} 1=0,0072$ y en la franja dos la tasa de descomposición $(k) f 2=$ 0,0081. La tasa de descomposición más baja se presentó en la franja uno (Figura 2).

Figura 2. Tasa de descomposición $(k)$ entre franjas.

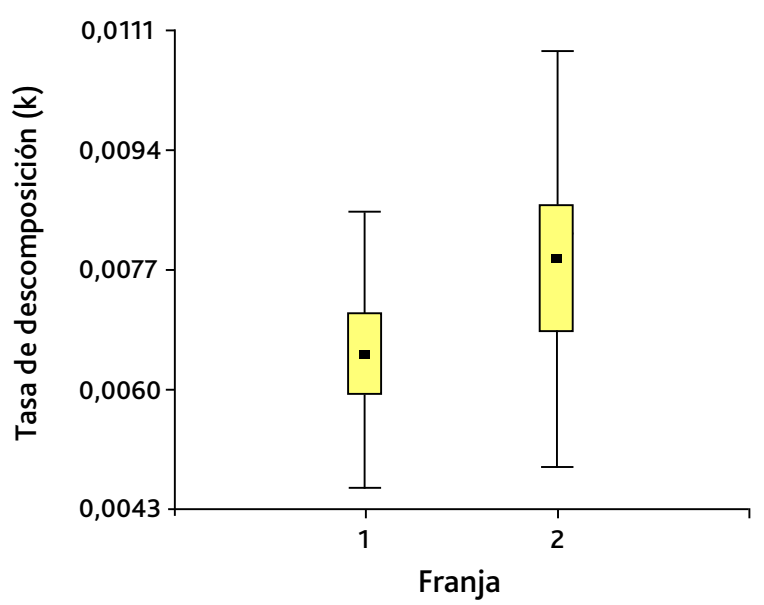

Fuente: Reyes, 2021. 


\section{Potencial uso}

La metodología implementada en esta investigación se puede replicar a bosques subandinos y urbanos con características climatológicas similares, permitiendo de esta forma establecer relaciones de conectividad entre matrices similares que contribuya a generar estrategias de conservación para mitigar la afectación antrópica.

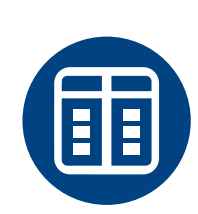

Ficha técnica del proyecto
Título del proyecto que lo deriva o vincula: Evaluación de la producción y descomposición de hojarasca en el remanente de bosque El Espejo de la Universidad de Ibagué.

PRIT: Conservación y aprovechamiento ecoeficiente de los recursos naturales y el medio ambiente.

Código del proyecto: N/A.

Palabras claves: Peso seco, bosque de ribera, peso remanente, servicios ecosistémicos.

Grupo de Investigación: Naturatu.

Investigador principal: Blanca Myriam Salguero Londoño.

Correo electrónico: blanca.salguero@unibague.edu.co

\section{Referencias}

Alvear, N., Betancur, J., \& Franco, P. (2010). Diversidad florística y estructura de remanentes de bosque andino en la zona de amortiguación del Parque Nacional Natural Los Nevados, cordillera central colombiana. Caldasia, 32(1), 39-63. Recuperado de https://revistas.unal.edu.co/index.php/cal/article/view/36193

Balvanera, P. (2012). Los servicios ecosistémicos que ofrecen los bosques tropicales. Ecosistemas, 21(1-2), 136-147. Recuperado de https://www.revistaecosistemas.net/index.php/ecosistemas/article/ view/33

Ceccon, E. (2003). Los bosques ribereños y la restauración y conservación de las cuencas hidrográficas. Ciencias, 72 (1), 46-53.

Díaz, J., \& Curiel, A. (2012). Bosques urbanos para enfriar las ciudades. Ciencia-Academia Mexicana de Ciencias, 63(4), 36-41.

Isaac, S., \& Nair, M. (2006) Litter dynamics of six multipurpose trees in a homegarden in Southern Kerala, India. Agroforestry systems, 67(3), 203-213.

Moll, G., \& Gangloff D. (1987). Silvicultura urbana en los Estados Unidos. Unasylva, 39(155), 26-32.

Naiman, R., Decamps, H., Mcclain, M., \& Likens, G. (2005). Riparian typology. En Riparia: ecology, conservation, and management of streamside communities (pp 49-78). London: Academic Press.

Reyes, A. (2021). Evaluación de la producción y descomposición de hojarasca en el remanente de bosque "El Espejo" de la Universidad de Ibagué (Tesis de pregrado). Universidad de Ibagué, Ibagué, Colombia.

Wider, R. \& Lang, G. (1982). A critique of the analytical methods used in examining decomposition data obtained from litter bags. Ecology, 63(6), 1636-1642. 